\title{
Specific Diversity of School Stochastic Problems
}

\author{
Andrey Aksenov ${ }^{1, a *}$, Vladimir Selutin ${ }^{1, b}$ \\ 1 Orel State University named after I. S. Turgenev, 30202695 Komsomolskaya str., Orel,
Russia
}

\author{
aaksenovaa@inbox.ru, bselutin_v_d@mail.ru \\ ${ }^{*}$ Corresponding author
}

Keywords: stochastics, probability, types of problems, problem solving

\begin{abstract}
In the modern theory and methodology of teaching mathematics, the theoretical basis for the application of problems in the learning process is developing. In particular, the traditional view on the species composition of tasks is also changing. Their new species are proposed. A few years ago, elements of the theory of probability and mathematical statistics were strongly included in the school course of mathematics, a significant part in the study of which consists of stochastic problems. These tasks should be not only an integral part of the stochastic educational material, but also get a full description in the general theory of school math problems. In particular, this article shows that stochastic problems fully satisfy the species diversity of problems in a school mathematics course.
\end{abstract}

\section{Introduction}

The elements of stochastics relatively recently took their place in the school course of mathematics, despite the fact that methodical preparation for this has been done for a long time, which follows, for example, from the paper [5]. The stochastic problems described quite fully in [4] are peculiar. At the same time, they can fully and should correspond to all theoretical and methodological characteristics of school mathematical problems (typology, types of problems, classes of problems, four-aspect typology of their theoretical basis, and many others. [1]), i.e. to become an integral part of the general theory of problems described in a number of papers $[1,2,3$, etc.]. In this article, we will show that stochastic problems fully meet the modern diversity of school math problems.

\section{Methodology}

The main methodological tools of the study are: dialectics; the genetic constructive method; the method of constructive introduction of abstract objects; methods of modeling, analysis, synthesis, induction, deduction, analogy, comparison, generalization, and concretization; the systems approach.

\section{Study}

Traditionally, in domestic high school there are three types of problems: problems for computing, problems for proof, and problems for construction. This kind of task diversity is based on the essence of the requirement (as a motivating sentence) contained in the statement of the problem. However, in school mathematics, there are problems whose condition is mainly given visually, such as a graph of a function (or its derivative), and it is necessary to describe verbally some peculiarities in the behavior of this function. Obviously, such a task clearly does not belong to any of the three types mentioned earlier. In addition, in a number of publications, scientists, methodologists, mathematicians, and teachers of mathematics point to tasks that, by their nature (due to the requirement in their formulation), imply research, and they are called research tasks, that is, they also offer the term (practically all tasks with parameters, etc.). Thus, the request for expanding the species spectrum of problems in school courses of mathematics was formed long ago.

In the modern methodology of teaching mathematics, it is advisable to single out seven main types of tasks: problems of finding; tasks for proof; construction tasks (in algebra these are graphing tasks); research tasks; constructive tasks (tasks for creating tasks that meet predetermined requirements); problems solved by giving a specific example or counterexample; problems solved by means of a verbal description [1]. Note that in this article, the term "task of finding" is used, and not the "task of calculation," because in 
many of these tasks, the desired is not represented by a specific number. It can also be represented by a literal expression, which was eventually found, often rather cumbersome and uncomplicated in the following. Therefore, the proposed problem is the task of finding with the aim of generalizing into one type of all tasks, in which the essence of the requirements is the same, but the presentation of the desired is slightly different.

Of course, for all seven selected types of tasks, the rule for determining the species belonging of a task remains in force: the ratio of mathematical tasks to a particular type is determined only by the essence of the requirement in them. Moreover, when defining the type of a task, it is necessary to take into account not the particularities of the editorial staff, its requirements, but its meaning, establishing which, in many cases, should be taken into account the vocabulary traditional for school math problems used in its formulation. Moreover, it should be borne in mind that in the extremely general case, the requirement of a task is uniquely related to it with this or that type of task only taking into account the possibility of performing it, which is characteristic of tasks of this type, and the fact that the task belongs to any type is determined only by this circumstance.

Let us show that stochastic problems can fully correspond to all these seven types of tasks.

Obviously, the majority of stochastic problems are related to the type of problems for finding (calculating); therefore, additional comments are superfluous here. The type of construction tasks (graphs) includes tasks in which it is required to plot the distribution function or density distribution function. The corresponding tasks in school mathematics are considered in specialized mathematical classes or on elective (or on optional) courses.

The type of problems solved by casting a specific example or a counterexample includes, for example, the following problem: can the probability of hitting a target after two shots be higher than 0.8 if the probability of hitting a target for each of the shooters does not exceed 0.9 ? The affirmative answer is illustrated by an example when both arrows hit the target and the probability of hitting for each of them is 0.9 , then the desired probability will be equal to 0.81 . Developing the topic of this problem, one can come, for example, to the next problem on the proof. Two arrows shoot at the target once. Prove that the maximum attainable probability of any of the possible events is always higher than the probability of hitting the target by one shooter. Let $\alpha$ be the probability of hitting the target first, $\beta$ is the second shooter, and $\alpha>$ $\beta$. The greatest probability of an event is that at least one hit in the target: $\alpha(1-\beta)+\beta(1-\alpha)+\alpha \beta$. We prove that $\alpha(1-\beta)+\beta(1-\alpha)+\alpha \beta>\alpha$. After conducting transformations, we get: $\beta(1-\alpha)>0$. Given that $\alpha$ and $\beta$ are positive and less than 1 , the inequality can be considered proven.

An example of a problem solved by a verbal description is the problem of the following content. A series of even numbers of games is played by two equal chess players. For each of them, the probability of winning: one batch of two is 0.5 ; two batches of four are equal to 0.375 ; three batches of six are equal to 0.3125 . Explain why the probability of winning half the games in the series decreases with an increase in their number, despite the equal strength of the players?

Constructive tasks are tasks for creating tasks with a number of specified features. With regards to constructive tasks, we refer, for example, to the following: "Create a problem of throwing a die twice, in which the probability of an even number of points is mentioned." Possible solutions: a) the die is thrown twice, to find the probability of an even number of points falling out; b) the die is thrown twice, to prove that the probabilities of even and uneven points are equal.

In the course of algebra, research problems are mainly represented by problems with parameters. Transforming the task 15 from [4, p. 169], we get a stochastic research task: "When throwing a coin on the table in $\beta \%$ of experiments, it rolled onto the floor; find event rates of the emblem and numbers if $\alpha \%$ of all eruptions and $30 \%$ of all the figures fell on the floor". Expressing the percentages by decimal fractions a and $\mathrm{b}(0 \leq \mathrm{a}, \mathrm{b} \leq 1)$ and denoting the desired by $\mathrm{x}$ and $\mathrm{y}(0 \leq \mathrm{x}, \mathrm{y} \leq 1)$, we obtain the following system of equations: $a x+0.3 y=b, x+y=1$. By rewriting this system in the form $y=-a x / 0.3+b / 0.3, y=-x+1$ and using graphical considerations (arranging two straight lines on a plane), we get this result after performing a simple solution. If $\mathrm{a}=\mathrm{b} \neq 0.3$, then $\mathrm{x}=1, \mathrm{y}=0$; if $\mathrm{a}=\mathrm{b}=0.3$, then $\mathrm{x}=\mathrm{t}, \mathrm{y}=1-\mathrm{t}$, where $0 \leq \mathrm{t}$ $\leq 1$; if $0.3<\mathrm{b}<\mathrm{a}$, or $\mathrm{a}<\mathrm{b}<0.3$ (and $0 \leq \mathrm{a}, \mathrm{b} \leq 1)$, then $\mathrm{x}=(\mathrm{b}-0.3) /(\mathrm{a}-0.3), \mathrm{y}=(\mathrm{a}-\mathrm{b}) /(\mathrm{a}-0.3)$. In all other cases, there are no solutions for $\mathrm{a}$ and $\mathrm{b}(0 \leq \mathrm{a}, \mathrm{b} \leq 1)$. 
In general, stochastics as a separate topic of school mathematics, studied by students for several years, are directly connected with almost all of its other topics, so there is a prospect of using stochastics as a tool to strengthen intra-subject relations in the process of teaching mathematics. In this regard, stochastic problems are the most effective means, since the theoretical material of probability theory and mathematical statistics still contains a number of specific aspects, and the subject material of stochastic problems can include educational material on almost any other topic. In the development of this idea, the concept of a coherent stochastic problem was introduced in the monograph [4], which is the main tool for strengthening intradisciplinary connections with the help of stochastics [4, c. 127-135].

In the modern methodology of teaching mathematics in the works of many scientists it is substantiated that the implementation of intra-subject relations in the process of teaching mathematics (primarily through problem solving) is one of the most effective tools for improving the quality of mathematical education of schoolchildren; therefore, the use of stochastic problems for these purposes seems quite appropriate. The expansion of the species diversity of stochastic problems can only contribute to the emergence of new opportunities for implementing and, consequently, strengthening intra-subject relations. Thus, applying stochastic problems of all seven types in the course of mathematics strengthens intradisciplinary connections in it.

We also note that the possibility of compiling school math problems of all seven types within the framework of educational material of any topic, in particular, stochastics, is in itself a realization of intradisciplinary connections through problem solving, since it demonstrates the possibility of combining one of the most important theoretical and methodological characteristics, - tasks (their type) with specific facts inherent in this topic.

\section{Conclusion}

The results presented in the article allow us to conclude that stochastic problems fully meet the entire species diversity of school math problems. That is, stochastic problems can and should be considered as part of the general theory of school mathematical problems, which, in turn, is an integral part of the integration of this theory and theory and methods of teaching stochastics. This, of course, strengthens the scientific basis of the methodology of teaching mathematics in school.

Types of stochastic problems have specialized didactic purpose. The problems of finding the most typical one are used in learning stochastics. Tasks solved with a specific example or a counterexample as well as constructive tasks can be used (for example, in extracurricular activities to increase interest in the subject). Evidence tasks and tasks solved by verbal description contribute to a deeper understanding of the essence of stochastic processes by students. The didactic assignment of tasks for plotting is obvious. The research tasks are rather difficult, but they can contribute to teaching pupils to perform stochastic interpretations of the obtained quantitative results, etc.

The species diversity of stochastic problems contributes to the expansion of the ability to control the knowledge of schoolchildren, i.e. one of the most important conditions for their quality education [7], which is a weighty argument for using tasks of all existing types in teaching stochastics. Note that, at present, the role of stochastics in school and after-school education in mathematics is only increasing, which is confirmed by contemporary publications [6]. Therefore, studying the essence of stochastic problems and their significance in the mathematical education is relevant and timely.

\section{References}

[1] Aksenov, A. A. (2010). Theory of teaching logical search for the solution of school mathematical problems: Diss.... Dr. Ped. Sciences: 13.00.02. Orel, Russia.

[2] Kolyagin, Yu. M. (1977). Tasks in teaching mathematics. Part I. Moscow, USSR: Enlightenment.

[3] Kolyagin, Yu. M. (1977). Tasks in teaching mathematics. Part II. Moscow, USSR: Enlightenment.

[4] Selyutin, V. D., \& Terekhova, L. A. (2008). Strengthening intradisciplinary connections of the school course of mathematics by means of stochastics: monograph. Orel, Russia: OGU. 
[5] Plotski, A. (1991). Mathematics in school, 3, 34-42.

[6] Chen, L., Davydov, Y., \& Gribkova, N. (2018). Estimating the index of increase via balancing deterministic and random data mathematical methods of statistics (vol. 27, No. 2, pp. 83-102). New York, NY: Allerton Press, Inc.

[7] Shikhnabieva, T., \& Beshenkov, S. (2016). Intelligent system of training and control of knowledge, based on adaptive semantic models. Smart Innovation, Systems and Technologies, 99, 84-93. 